\title{
Comprehensive circular RNA profiling reveals that circular RNA100783 is involved in chronic CD28-associated CD8(+)T cell ageing
}

Yu-hong Wang ${ }^{1,2}$, Xu-hui Yu ${ }^{3}$, Shan-shun Luo ${ }^{1,2}$ and Hui Han ${ }^{1,2^{*}}$

\begin{abstract}
Background: Ageing brings about the gradual deterioration of the immune system, also known as immunosenescence. The role of non-coding circular RNA in immunosenescence is under studied. Using circular RNA microarray data, we assembled Comparison groups (C1, C2, C3 and C4) that allowed us to compare the circular RNA expression profiles between CD28(+)CD8(+) T cells and CD28(-)CD8(+) T cells isolated from healthy elderly or adult control subjects. Using a step-wise biomathematical strategy, the differentially-expressed circRNAs were identified in C1 (CD28(+)CD8(+) vs CD28(-)CD8(+)T cells in the elderly) and C4 (CD28(-)CD8(+)T cells in the elderly vs in the adult), and the commonly-expressed circRNA species from these profiles were optimized as immunosenescence biomarkers.

Results: Four overlapping upregulated circular RNAs (100550, 100783, 101328 and 102592) expressed in cross-comparison between C1 and C4 were validated using quantitative polymerase chain reaction. Of these, only circular RNA100783 exhibited significant validation. None of the down-regulated circular RNAs were expressed in the $\mathrm{C} 1$ and the C4 cross-comparisons. Therefore, we further predicted circular RNA100783-targeted miRNA-gene interactions using online DAVID annotation. The analysis revealed that a circular RNA100783-targeted miRNA-mRNA network may be involved in alternative splicing, the production of splice variants, and in the regulation of phosphoprotein expression. Considering the hypothesis of splicing-related biogenesis of circRNAs, we propose that circular RNA100783 may play a role in phosphoprotein-associated functions duringCD28-related CD8(+) T cell ageing.

Conclusions: This study is the first to employ circular RNA profiling to investigate circular RNA-micro RNA interactions in ageing human CD8(+)T cell populations and the accompanying loss of CD28 expression. The overlapping expression of circular RNA100783 may represent a novel biomarker for the longitudinal tracking ofCD28-related CD8(+) T cell ageing and global immunosenescence.
\end{abstract}

Keywords: T cell, Ageing, CD28, Circular RNA, Microarray, Biomathematics

\section{Background}

The ongoing loss of immune function during ageing, or immunosenescence, is a major contributor to the increasing morbidity and mortality in the elderly population. One of the prominent alterations on immune cells during this

\footnotetext{
* Correspondence: hydhanhui@sina.com

Xu-hui Yu contributed as the co-first author.

'Department of Geriatrics, First Affiliated Hospital of Harbin Medical

University, Harbin 15001, China

${ }^{2}$ First Institute of Geriatrics and Gerontology of Harbin Medical University,

Harbin 15001, China

Full list of author information is available at the end of the article
}

process is the gradual loss of the co-stimulatory molecule, CD28, from the surface of CD8(+) T lymphocytes $[1,2]$. Thus, an increase in the CD28(-)CD8(+) T subset is considered to be one hallmark of immunosenescence [3]. However, except for their replicative characteristics during senescence $[4,5]$, very little is known regarding the exact nature, origin and function of CD28(-)CD8(+)T cells [1]. Indeed, the mechanism by which $\mathrm{CD} 28$ is lost from CD28(+)CD8(+)T cell surface is complicated. During an individual's lifespan, CD28(+)CD8(+)T cells are gradually stimulated by encounters with numerous antigens resulting in CD28 exhaustion. Latent human cytomegalovirus 
(CMV) is responsible for the greatest clonal expansion of CD28(-)CD8(+) $\mathrm{T}$ cells in the healthy elderly population [6-9]. Latent CMV carriers are so prevalent that CMV seropositivity has been proposed as one of the "Immune Risk Profiles (IRPs)", and individuals in the general elderly population who exhibit high IRP have an increased risk of all-cause mortality [10,11]. Moreover, microarray analyses of elderly and adult control subjects revealed that 754 genes representing a wide range of biological processes and cellular functions are significantly altered in human CD8(+) $\mathrm{T}$ cells [12]. It has been observed that CD28(-)CD8(+) T cells demonstrated similar patterns of gene expression in elderly and adult subjects $[13,14]$, while the profiles of gene expression in CD28(+)CD8(+) T cells are profoundly altered in adult and the elderly populations [14]. However, the disparities between adult and old, as well as between CD28(-)CD8(+) and CD28(+)CD8(+)T cells, remain unexplored.

More than 25,000 species of an enigmatic class of circular RNAs have been identified in various human cells [15]. At least two circular RNAs have been demonstrated to function as molecular sponges to their target miRNAs $[16,17]$. It has been hypothesized that circular RNAs are produced by non-random back-splice events that forma covalently closed continuous loop [18], and some generalized mechanism might strictly determine whether splicing produces a circular non-coding RNA or a linear mRNA [19]. The expression of circRNA was found to be tissue- as well as developmentally-specific $[17,20]$. The levels of circular RNAs also increase in an age-related manner in Drosophila [21]. Until the present study, age-related circular RNA profiling in lymphocytes, and the circRNA-dependent molecular regulation ofCD28 exhaustion from the surface of $\mathrm{CD} 8(+) \mathrm{T}$ cell, has remained under studied.

The development of circRNA microarray (http:// www.kangchen.com.cn/english/) has facilitated the study of the role of circular RNAs in regulating gene expression through a circRNA-miRNA-mRNA network. To explore the underlying molecular regulation of circRNAs in T cell ageing, we first used circular RNA microarray detection to acquire circRNA profiles in CD28-dependent CD8(+)T cell subsets in elderly individuals(Q1 and Q2) and adult controls (Q3 and Q4). Based on it, we compared cross them to acquire four cross-differentiated circular RNA profiles $(\mathrm{C} 1, \mathrm{C} 2, \mathrm{C} 3$ and $\mathrm{C} 4)$. Then we performed stepwise bioinformatics analysis to identify the overlapping circular RNA molecules between $\mathrm{C} 1$ and $\mathrm{C} 4$ as potential biomarkers of $\mathrm{CD} 8+\mathrm{T}$ cell immunosenescence.

\section{Results and discussions}

Clinical characteristics of the enrolled subjects

We enrolled in our study a total of 21 apparently healthy elderly individuals and 8 healthy adult volunteers, who had been initially identified as CMV latent carriers by CMV-IgG detection. The general clinical characteristics of the 29 participants are shown in Table 1.

\section{General profiles of circRNA microarray}

Overall, 2171 circRNAs were significantly up-regulated among the four comparison groups $\left(\mathrm{N}_{\mathrm{Cl}(\mathrm{Q} 1 \mathrm{vs} \mathrm{Q} 2)}=478\right.$, $\left.\mathrm{N}_{\mathrm{c} 2(\mathrm{Q} 3 \mathrm{vs} 4)}=272, \mathrm{~N}_{\mathrm{C} 3(\mathrm{Q} 1 \mathrm{vs} \mathrm{Q} 3)}=671, \mathrm{~N}_{\mathrm{C} 4(\mathrm{Q} 2 \mathrm{vs} 4)}=741\right)$. (For the concise aim, we abbreviated circular RNA as "circRNA" in the following manuscript). In these same groups, 1563 circRNAs were significantly down-regulated $\left(\mathrm{N}_{\mathrm{C} 1\left(\mathrm{Q} 1 \mathrm{vs} \mathrm{Q}_{2}\right)}=\right.$ $\left.337, \mathrm{~N}_{\mathrm{c} 2(\mathrm{Q} 3 \mathrm{vs} \mathrm{Q} 4)}=332, \mathrm{~N}_{\mathrm{C} 3(\mathrm{Q} 1 \mathrm{vs} \mathrm{Q} 3)}=438, \mathrm{~N}_{\mathrm{C} 4(\mathrm{Q} 2 \mathrm{vs} \mathrm{Q} 4)}=456\right)$. We ranked the circRNAs according to fold-change (FC) in expression levels of the 4 comparison profiles and listed the Top10 candidates ("Top 10") of each comparison in Additional file 1: Table S1.

\section{Identification of Top-5 miRNAs according to mirSVR}

For each circRNA with fold changes in expression levels greater than 2 in the four comparison groups, we ranked their targeted miRNAs according to mirSVR scores and identified the 5 highest ranking candidates ("Top 5") further analysis (for details see Additional file 2: Online Supplemental Excel 1). We then utilized Cytoscape to construct circRNA-miRNA networks of interaction between those circRNA exhibiting a 2-fold or greater change in expression (up-regulated and down-regulated expression) and their Top 5 targeted miRNAs (designated as "Top-5 network") (Additional file 3: Figure S1). Since the number of targeted miRNAs that interplayed with each circRNA in these two networks was almost equal $\left(\mathrm{N}_{\text {miRNA }}=5\right)$, the profiles of these two networks appeared to be similar. There were more molecular interactions in the down-regulated Top-5network (2338 nodes with 7723 edges) than in the up-regulated Top-5 network (1865 nodes with 5558 edges).

Identification of overlapping circRNAs between $\mathrm{C} 1$ and $\mathrm{C} 4$ Until now, the molecular interactions between most circRNAs and their target miRNAs have been addressed by theoretical prediction based on the principle of baseparing. Moreover, for any one circRNA, at least more than ten miRNAs have been predicted as potential targets. Therefore, it can be challenging to define which circRNA molecules play more critical or pivotal roles in a complex circRNA-miRNA network. Here, we supposed that circRNAs that interact with more miRNA targets play a more significant role in the general circRNA-miRNA network, than do those circRNAs that interact with fewer targets. Based on this hypothesis, we designed a step-wise strategy to optimize potential candidate circRNAs.

Firstly, among the up-regulated and down-regulated Top-5 networks, the Top- $10 \%$ miRNAs ranked by 
Table 1 The clinical characteristics of the subjects with CMV seropositivity

\begin{tabular}{|c|c|c|c|c|}
\hline \multirow[t]{2}{*}{ Category } & \multicolumn{3}{|l|}{ Elderly group } & \multirow[t]{2}{*}{ Adult group } \\
\hline & Old-old & Middle-old & Adult-old & \\
\hline Number & 6 & 7 & 8 & 8 \\
\hline Age & $90.5 \pm 2.4$ & $79.6 \pm 2.5$ & $69.3 \pm 3.1$ & $32.0 \pm 4.6$ \\
\hline Gender (male vs female) & $3 v s 3$ & $4 \mathrm{vs} 3$ & $4 \mathrm{vs} 4$ & $4 \mathrm{vs} 4$ \\
\hline Number of chronic diseases ${ }^{a}$ & 3.4 & 3.0 & 2.9 & 0 \\
\hline Number of daily medications(NNTs) ${ }^{b}$ & 5.6 & 5.3 & 5.1 & 0 \\
\hline Numbers of white blood cell $\left(10^{9} \mathrm{~L}^{-1}\right)$ & $7.14 \pm 3.67$ & $7.09 \pm 3.56$ & $7.20 \pm 2.98$ & $7.98 \pm 3.52$ \\
\hline Numbers of $\mathrm{PBMC}\left(10^{9} \mathrm{~L}^{-1}\right)$ & $0.44 \pm 0.25$ & $0.43 \pm 0.26$ & $0.44 \pm 0.24$ & $0.48 \pm 0.21$ \\
\hline Numbers of $\mathrm{CD} 8(+)\left(10^{9} \mathrm{~L}^{-1}\right)$ & $0.26 \pm 0.14$ & $0.23 \pm 0.12$ & $0.25 \pm 0.15$ & $0.24 \pm 0.11$ \\
\hline Percentage ratio of CD28(+)/CD28(-) & $43.4 \pm 22.1 / 56.6 \pm 33.5$ & $49.1 \pm 26.5 / 57.1 \pm 37.8$ & $46.9 \pm 22.1 / 53.1 \pm 39.0$ & $55.1 \pm 29.2 / 42.6 \pm 29.3$ \\
\hline C-reactive Protein $\left(\mathrm{mg} \mathrm{L}^{-1}\right)$ & $6.39 \pm 2.86$ & $6.26 \pm 3.02$ & $6.41 \pm 3.27$ & $5.22 \pm 1.45$ \\
\hline
\end{tabular}

${ }^{a}$ Number of chronic disease (multimorbidity) was calculated using 10 age-related/chronic diseases including sustained/primary hypertension, primary hyperlipidemia, type 2 diabetes, coronary atherosclerotic heart disease, chronic atrial fibrillation, preclinical valvular disease, preclinical chronic obstructive pulmonary disease, subclinical chronic liver disease, subclinical chronic renal disease, subclinical thyroid disease and long-term(subclinical $>5$ years) tumor survivor

${ }^{\mathrm{b}}$ Number of daily medications were calculated by the number of drugs necessary to treat (NNTs). It was calculatedusing 15 categories of pharmaceutical administration,including:5 oral antihypertensive drugs (angiotensin receptor blockers, angiotensin-converting enzyme inhibitors, calcium channel blockers, beta-receptor blockers, and hydrochlorothiazide); 4 oral hypoglycemic drugs (sulfonylureas, metformin, glucosidase inhibitors, and thiazolidinediones); insulin or insulin analogues;

2 lipid lowering drugs(statin and fibrate); aspirin; clopidogrel; trimetazidine; CoenzymeQ10; and isosorbidemononitrate sustained-release capsules

miRNA-dependent degree (D) were optimized for next step. In the re-ranked Top-10\% up-regulated network, 75 up-regulated miRNAs (Additional file 1: Table S3-1) with degree values ranging from 22 to 60 were selected. The highest degree of miRNA was hsamiR-136-5p $(D=60)$, indicating that it interplayed with 60 different circRNAs in this network. In the reranked Top-10 \% down-regulated network, 80 miRNAs with degree value ranging from 28 to 81 were selected (Additional file 1: Table S3-2). The miRNA hsa-miR608 exhibited the highest degree $(\mathrm{D}=81)$, indicating that it was regulated by 81 different circRNAs in this network. The distribution of these Top-10 \% miRNAs is depicted in Additional file 3: Figure S2. Using these Top-10\% miRNAs, up-regulated and down-regulated miRNA-circRNA networks (designated as Top-10\% network-mi) were re-enriched on the basis of the fundamental miRNA-circRNA connectivity (Additional file 3: Figure S3). The distribution of degree of the miRNAs of the re-enriched Top-10\%-mi network is depicted in Additional file 3: Figure S4.

Then, we re-ranked the Top-10\% network according to circRNA-mediated degree (designated as Top10\%-network-ci). In this re-ranked data set, the degree of up-regulated circRNAs ranged from 1 to 12. The circRNA100550 exhibited the largest degree $(\mathrm{D}=12)$, indicating that it interplayed with $12 \mathrm{miR}$ NAs. The degree of down-regulated miRNAs ranged from 1 to 15 . The circRNA100988 exhibited the largest degree $(D=15)$, indicating that it interacted with 15 miRNAs. The distribution of the degree of the circRNAs of the Top-10\%-ci network is depicted in
Additional file 3: Figure S5. From the figure, we could observe that a majority of the circRNAs interacted with only one or two miRNAs (237 circRNAs of $\mathrm{D}=1$ and 307 circRNAs of $\mathrm{D}=2$ ). In order to optimize intracellular validation analyses, we selected a subset of circRNAs of the largest Degree as the candidates. Here, we optimized our parameters to select circRNAs with degree values greater than 9 (Top Degrees, $D \geq 9$ ) and enrolled the 12 up-regulated circRNAs and the12 downregulated circRNAs with the highest degree values for intracellular validation studies.

\section{Venn analysis for overlapped circRNA identification by crosstalk analysis}

To determine which circRNAs exhibited overlapping expression between the $\mathrm{C} 1$ and $\mathrm{C} 4$ comparison groups, Venn analyses were performed using the selected 12 upregulated and 12 down-regulated circRNAs (The Venn's diagram is depicted in Additional file 3: Figure S6). The details of between-group comparisons are also shown in Table 1. Four of twelve up-regulated circRNAs were expressed in both the $\mathrm{C} 1$ and $\mathrm{C} 4$ groups. Therefore, we employed $\mathrm{qPCR}$ to validate the intracellular expression of these four circRNAs in their respective $T$ cell subsets of origin. Unexpectedly, none of the 12 candidate circRNAs mediated crosstalk in the down-regulated network. To compare the same number of circRNAs/ molecules as in the up-regulated network, we selected four circRNAs $(\mathrm{N}=4$, Top-2 Fold Changes in $\mathrm{C} 1$ or $\mathrm{C} 4$, respectively) for the intracellular validation in the downregulated network. 


\section{Cellular validation using $\mathrm{qPCR}$}

The relative intracellular expression (ratio of intracellular expression to microarray expression) of the aforementioned 8 circRNAs is shown in Fig. 1. At the intracellular level, we found that only circRNA100783 expression significantly differed between the $\mathrm{C} 1$ and $\mathrm{C} 4$ groups. This indicated that the expression of circRNA100783 was affected by time (C4) and antigen exposure (C1) during
CD28-related CD8(+)T cell ageing. Therefore, we propose that this circRNA100783 is a potential biomarker in ageing $\mathrm{T}$ lymphocytes.

\section{circRNA100783-targeted miRNA-mRNA network prediction} and annotation

According to the initial array data analysis, the genomic locus of circRNA100783 is on chromosome 11and the

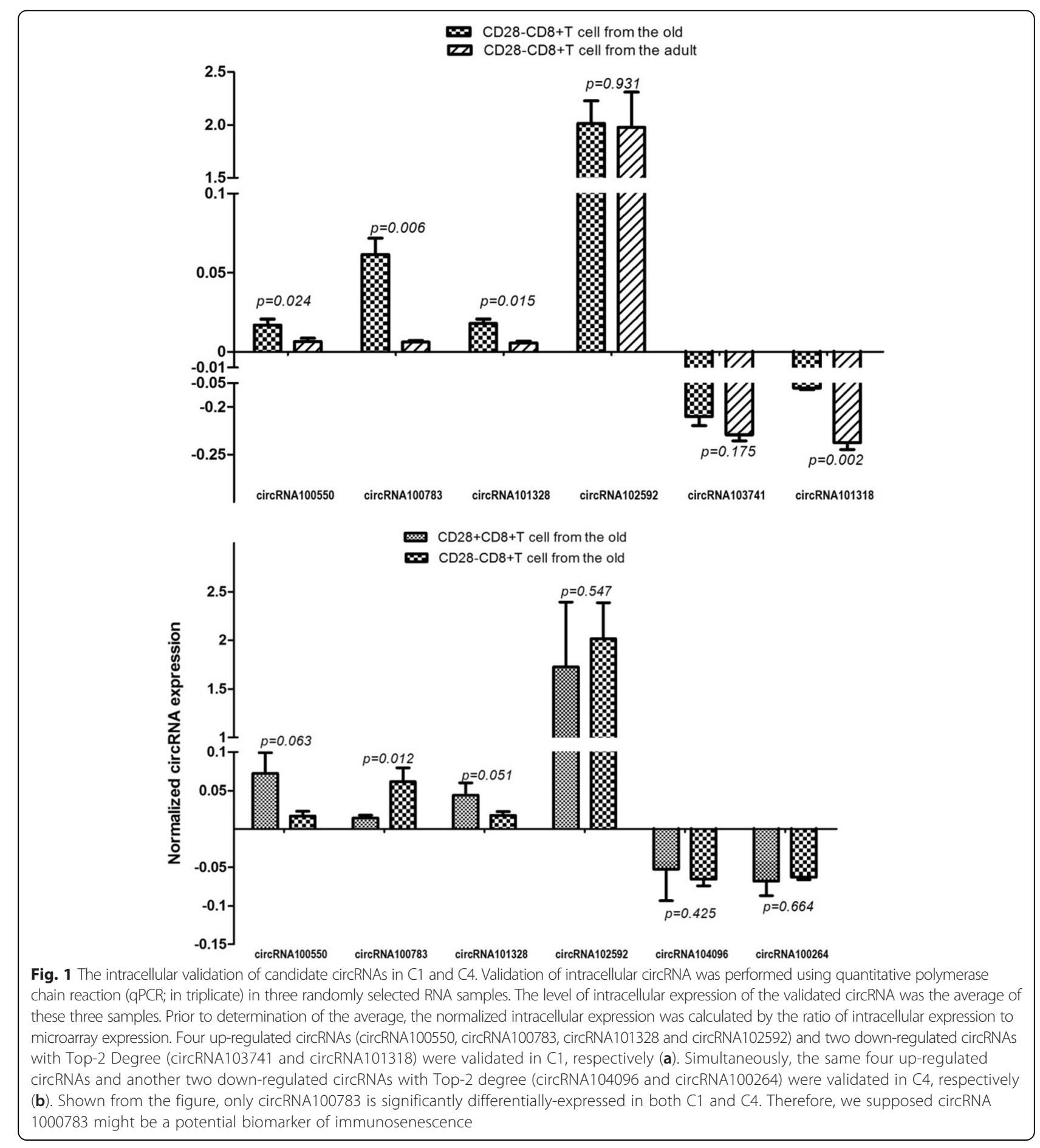


predicted sequence of the best linear transcript isuc001mul.1. The molecular interaction of circRNA100783 with its Top-5 miRNA targets is depicted in Additional file 1: Table S4. Supposing that circRNA100783 is an upstream molecular sponge in its circRNA-miRNA-mRNA network, we predicted a circRNA100783-miRNA-gene network using Targetscan and miRanda. A total of Top18 miRNAs (miRNA-dependent cutoff value -0.11) andTop-6 miRNAs (mRNA-dependent cutoff value -0.38) were finally determined in this network (Fig. 2). Using DAVID function annotation, circRNA100783 was predicted to be associated most strongly with alternative splicing, as well as with splice variation and phosphoprotein function (Additional file 3: Figure S7). Given the strong correlation of the biogenesis of circRNAs with splicing and splice variation, it is possible that circRNA100783 functions in the regulation of a phosphoprotein that is involved in a molecular mechanism that leads to the age-related phenotypic loss of CD28 from CD8(+) T cells.

In the present study, we employed high-throughput circRNA microarrays to construct profiles of differentially expressed circRNAs in CD28-associated CD8 $(+) \mathrm{T}$ cells in the elderly and adult subjects $(\mathrm{C} 1, \mathrm{C} 2, \mathrm{C} 3$ and $\mathrm{C} 4)$. Then we used a step-wise bioinformatics protocol to optimize the Top differentially expressed candidate circRNAs, and then identify those circRNAs having expression profiles that overlap between the CD28(+)CD8(+)T cells and CD28(-)CD8(+)T cells from elderly donors (C1), as well as between the CD28(-)CD8(+)T cells from elderly and adult controls (C4). To narrow the range of the candidates we employed a step-wise optimizing strategy that consisted of ranking of RNAs (circRNAs or miRNAs) by degree values, based on their circRNA-miRNA molecular connectivity. Finally, the commonly expressed candidate circRNAs in $\mathrm{C} 1$ and $\mathrm{C} 4$ were validated in their respective $\mathrm{T}$ cell populations. Among these, the up-regulated circRNA100783 was the only candidate molecule that appeared to play both antigen-dependent $(\mathrm{C} 1)$ and agedependent (C4) roles in $\mathrm{CD} 8(+) \mathrm{T}$ cells during ageing. Therefore, circRNA100783 may serve as a novel biomarker of immunosenescence.

Several sources of heterogeneity could hamper ageing studies and we designed our analysis to minimize this pitfall. First, "absolute" healthy seniors were rare, while the common elderly population was affected with numerous chronic diseases (multimorbidity). Moreover, the increased heterogeneity of clinical phenotypes in the elderly was also correlated to age. To minimize this clinical heterogeneity, we enrolled representative elderly patients characterized as having "continuously asymptomatic manifestation" or "apparent health", the phenomena of which are rather prevalent in the general population. Second, over a lifetime, individuals may be exposed to several types of antigens that contribute to $\mathrm{T}$ cell immunosenescence. One characteristic phenotype is the eventual loss of CD28 from the CD8(+) T cell surface (CD28 exhaustion).

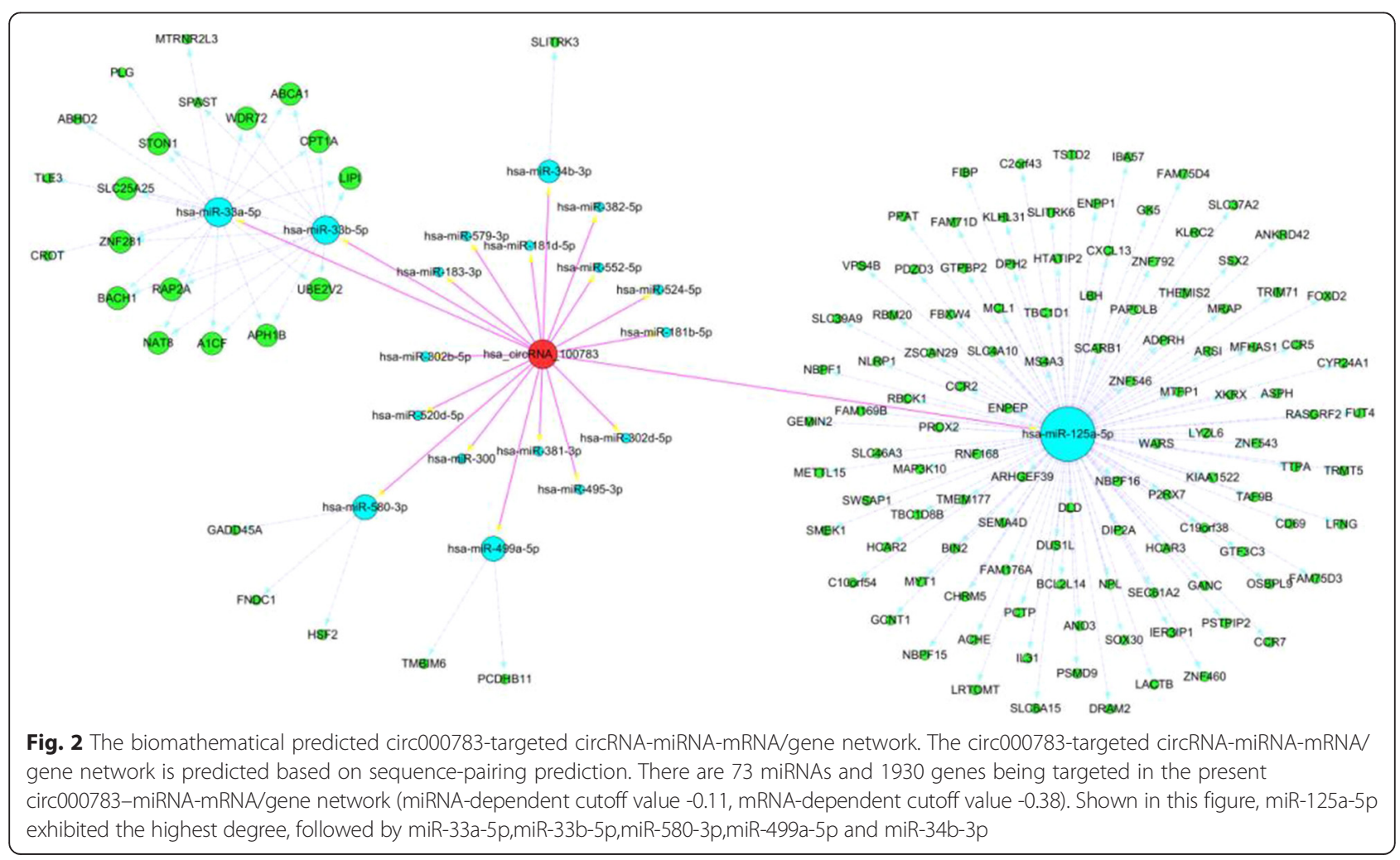


Cytomegalovirus (CMV) antigens are believed to elicit the greatest clonal expansion of CD8(+) T cells in healthy individuals during ageing $[6,7,22]$. Moreover, infection by CMV results in changes in gene expression in the aged immune system that are not observed in CMV-negative individuals [23]. Thus, to diminish the between-antigen heterogeneity, we limited our studies to subjects who tested seropositive for CMV according to microarray detection. Third, we examined samples of $\mathrm{CD} 28(+) \mathrm{CD} 8(+) \mathrm{T}$ cells and CD28(-)CD8(+) T cells from the same individuals, as well as among the elderly and controls groups.

A large amount of RNA is required to perform circRNA microarray analysis. However, even after increasing the blood sample size to $50 \mathrm{~mL}$, we could not isolate a sufficient amount of RNA from CD28(+)/(-)CD8(+) T cell subsets collected from elderly subjects. We propose that either the number of CD8(+)T cells or the total RNA from each cell, or both, decreased during ageing. Typically, cellular circRNAs are in relatively low abundance compared to their linear counterparts [24] and, therefore, we found it necessary to pool total RNA from several donors into one sample. To avoid potential molecular interaction, we constructed age-related subgroups of old donors, instead of using a random pooling protocol, to decrease the between-individual heterogeneity in the elderly group. Consequently, the elderly group was partitioned into three age-dependent subgroups: the adult-old subgroup (aged 65-74, $\mathrm{n}=8$ ); the middle-old subgroup (aged 75-84, $\mathrm{n}=7$ ) and the old-old subgroup (aged 85-94, $\mathrm{n}=6$ ).

Although 7,112 human circRNAs have been identified, and are estimated to comprise at least $10 \%$ of the transcripts accumulating from their loci, most circRNAs are expressed in only a few cell types and in low abundance [25]. Based on the current literature, it is unclear if, and to what extent, circRNAs regulate target miRNAs through sequence pairing. The elucidation of the molecular mechanism of circRNA-miRNA interactions would require numerous and extensive binding studies. A previous report showed that low levels of circRNAs may not be sufficient to affect the target molecules (Denzler et al. 2014). In view of this, we supposed that most circRNAs in the integrated circRNA-miRNA network played weak and transient interactions. Therefore, we designed a stepwise biomathematical protocol to identify candidates among differentially-expressed circRNAs based on their connectivity to target miRNA (independent of fold change). We considered that circRNAs which exhibited greater connectivity to miRNAs than did other circRNAs were more likely to play a role in the CD28 loss from CD8(+) $\mathrm{T}$ cell surfaces during ageing. After a basic circRNA-miRNA connectivity network was constructed, a step-by-step biomathematical analysis was performed.

The strategy we developed to analyze the expression of candidate circRNA consisted of three steps: 1) ranking theTop-5 miRNA targets in the circRNAmiRNA network and selecting the Top-10\% miRNAs according to miRNA-mediated connectivity to construct the Top-10 \%-mi network, 2) re-ranking the Top-10 \%-mi network according to circRNA-mediated connectivity to obtain a Top-10 \%-ci network and, 3) identifying candidate circRNAs with a degree value of 9 or greater in the Top-10 \%-ci network. Using the step-wise analysis, twelve up-regulated circRNAs and down-regulated circRNAs were identified as candidates for intracellular validation. We then employed a Venn diagram to explore which of these circRNA molecules exhibited overlapping expression in both the $\mathrm{C} 1$ and $\mathrm{C} 4$ comparison groups (Additional file 3: Figure S6). This permitted us to visualize changes in expression due to antigen-accumulated alterations (C1) and age (4). As shown in Table 1 (upper panel), a total of twelve up-regulated candidate circRNAs were distributed in the four comparison groups. Of the six up-regulated circRNAs from $\mathrm{C} 1$ and ten up-regulated circRNAs from C4, four molecules (circRNA100550, circRNA100783, circRNA101328 and circRNA102592) exhibited overlapping expression in the two groups. We subsequently validated these circRNAs in the corresponding $\mathrm{T}$ cell subsets using $\mathrm{qPCR}$. However, none of the down-regulated circRNAs were expressed in the four comparison groups (Table 2, lower panel). Therefore, we selected for validation two circRNAs which ranked in the Top-2, according to Fold Change, in the two comparison groups. Among these four up-regulated overlapping circRNAs, only the differential expression of circRNA101318 was validated by intracellular qPCR detection.

Until now, only two circRNAs have been functionally verified as miRNA sponges among thousands of detected circRNAs with predicted miRNA binding sites $[16,17]$. However, studies of SNP density at miRNA seed sites in 3 ' untranslated regions and flanking sequences, as well as at random sites, suggest that many of the predicted miRNA binding sites in circRNAs are functional and under similar selective pressure as are miRNA binding sites in mRNAs [26]. In the present study, we also predicted that circRNA100783 may function as a "sponge" to its targeted miRNAs in the predicted circRNAmiRNA-mRNA network. Then we used DAVID bioinformatics resources (Huang da et al., 2009) and KEGG pathway analysis (Kanehisa et al., 2006) to functionally annotate the predicted target genes of circRNA100783. According to our annotation, circRNA100783 is predicted to be involved in three possible functions including alternative splicing, splice variation and association with phosphoprotein(s) (Additional file 3: Figure S7).

\section{Conclusions}

In the present study, we used the cross-comparison of circRNA microarrays and step-wised bioinformatics to 
Table 2 The microarray Fold Change of the candidate circRNAs that were step-wise selected

\begin{tabular}{|c|c|c|c|c|c|}
\hline circRNA & Degree & C1:Q1 vs Q2 & C4:Q2 vs Q4 & C2:Q3 vs Q4 & C3:Q1 vs Q3 \\
\hline Up-regulated circRNAs & & Fold Change & & & \\
\hline hsa_circRNA_100550 & 12 & 2.1051912 & 2.7916539 & 4.9934264 & \\
\hline hsa_circRNA_001259 & 10 & 2.9541118 & & & 4.9224802 \\
\hline hsa_circRNA_101635 & 10 & & 2.6649119 & & 2.339966 \\
\hline hsa_circRNA_102592 & 10 & & 2.1983682 & & 2.1898953 \\
\hline hsa_circRNA_100315 & 10 & & 3.8537081 & 2.9419454 & \\
\hline hsa_circRNA_101358 & 9 & & 5.5122991 & 2.8866116 & 3.6304114 \\
\hline hsa_circRNA_101384 & 9 & & 10.8204701 & 2.7116116 & 3.3926842 \\
\hline hsa_circRNA_102592 ${ }^{a}$ & 9 & $\underline{3.8876708}$ & $\underline{2.7185627}$ & & 6.3889501 \\
\hline hsa_circRNA_400075 & 9 & & 6.9227289 & 5.663918 & 4.0526795 \\
\hline hsa_circRNA_104981 & 9 & 4.8466812 & & & \\
\hline hsa_circRNA_100783 & 9 & 3.1348047 & $\underline{4.1833976}$ & 7.213918 & \\
\hline hsa_circRNA_101328 & 9 & 2.2217774 & 2.2872726 & & 2.6027261 \\
\hline Down-regulated circRNAs & & Fold Change & & & \\
\hline hsa_circRNA_100988 & 15 & & 5.6605839 & 2.086644 & 2.938461 \\
\hline hsa_circRNA_100989 & 12 & & & 5.4144951 & 2.6900132 \\
\hline hsa_circRNA_103801 & 10 & & 2.1387435 & 3.3525614 & \\
\hline hsa_circRNA_104646 & 10 & 2.5271826 & & 2.1783708 & \\
\hline hsa_circRNA_100807 & 10 & & 2.4750588 & 3.7937131 & \\
\hline hsa_circRNA_101318 & 10 & & 7.3547169 & 6.3808594 & 3.0753515 \\
\hline hsa_circRNA_100264 ${ }^{\mathrm{b}}$ & 10 & 4.3266365 & & & 3.2787265 \\
\hline hsa_circRNA_103741 ${ }^{c}$ & 10 & & 7.7727788 & 2.5595041 & 3.8058778 \\
\hline hsa_circRNA_103572 & 10 & 3.2713424 & & & \\
\hline hsa_circRNA_104096 b & 10 & 3.2940966 & & & 2.0907926 \\
\hline hsa_circRNA_104266 & 9 & & 4.5747819 & 2.0283626 & 2.3366408 \\
\hline hsa_circRNA_103623 & 9 & 2.1655964 & & & 2.2533911 \\
\hline
\end{tabular}

aUp-regulated overlapping circRNAs of C1 and C4 (FC underlined)

bown-regulated circRNAs with Top-2 FC ofC1

'Down-regulated circRNAs with Top-2 FC of C4

Note: $\mathrm{C1:Q1}$ vs Q2 (CD28(+)CD8(+)T cells vs CD28(-)CD8(+)T cells in the elderly); $\mathrm{C2:Q3}$ vs Q4 (CD28(+)CD8(+)T cells vs CD28(-)CD8(+)T cells in the adult); C3=Q1 vs Q3 (CD28(+)CD8(+)T cells in the elderly vs the adult); $C 4=$ Q2vsQ4 (CD28(-)CD8(+)T cells in the elderly vs the adult)

determine that circRNA100783 may be involved in the loss of CD28 in CD8(+)T cells during ageing. A circRNAmiRNA-gene network suggests that circRNA100783, might regulate phosphoprotein-related signal transduction on CD28-dependent CD8(+)T cell ageing. A longitudinal study may verify circRNA100783 as a potential intracellular biomarker of immunosenescence.

\section{Methods}

\section{Participants}

The elderly donors were chosen from a group of participants in the Longitudinal Project on Witnessing Ageing (LPWA) in the Geriatrics Department of the First Affiliated Hospital of Harbin Medical University [27]. Each individual's annual medical documentation, including chronic disease, long-term clinical complaints, physical examinations, laboratory measurements, imageological diagnoses, daily life style, daily necessary medication, and pharmaceutical adjustments were comprehensively reviewed. Subjects aged 65 to 94 who were continuously asymptomatic in the recent twelve months were initially enrolled as "apparently healthy elderly individuals". After testing for latent human cytomegalovirus (CMV) infection by CMV-IgG, the CMV- seronegative subjects were excluded from the study. Adult CMV-seropositive healthy donors aged 25-35, who volunteered in the Geriatric Department, were enrolled and assessed by medical examination. All participants agreed to participate in the study with written content. The study was approved by the First Affiliated Hospital of Harbin Medical University of Medicine Ethic Committee and the research was carried out in compliance with the Helsinki Declaration. 
Isolation of CD28(+)CD8(+) T cells and CD28(-)CD8(+)T cells by flow cytometry sorting

A $20 \mathrm{~mL}$ intravenous blood sample was taken from each participant in the morning. Peripheral blood mononuclear cells (PBMCs) derived using Ficoll-Hypaque Plus gradient centrifugation were incubated in fluorescence-activated cell sorter (FACS) buffer with anti-CD8-phycoerythrin (PE; BD Biosciences) and anti-CD28-fluorescein isothiocyanate (FITC; BD Biosciences) for $30 \mathrm{~min}$. Then PBMCs were washed in FACS buffer, resuspended in $1 \%$ paraformaldehyde in FACS buffer, and sorted in a FACSCanto flow cytometer (BD Biosciences) in the National Key Laboratory of Harbin Vegetarian Research Institute. CD28(-)CD8(+)T cells and CD28(+)CD8(+)T cells from each donor were collected using two sterile collection tubes, respectively, for RNA extraction and analyses.

\section{circRNA microarray hybridization}

Total RNA from either CD28(-)CD8(+)T cells or CD28(+)CD8(+)T cells from the same subject was extracted and quantified using the NanoDrop ND-1000. RNA was prepared and stored at $-80{ }^{\circ} \mathrm{C}$ for the cellular validation. RNA from each sample was subjected to microarray analysis and hybridization according to the manufacturer's protocol (Arraystar). An aliquot consisting of $10 \%$ of the RNA from each sample was reserved for qRNA analysis.

\section{circRNA microarray data analysis}

Eight RNA samples extracted from either CD28(+)CD8(+) $\mathrm{T}$ cells or CD28(-)CD8(+) T cells were pooled into one RNA sample for one microarray detection (i.e. eight microarrays were performed). Then, data from 6 microarrays using RNA isolated from the elderly group were pooled into groups Q1 (CD28(+)CD8(+)) or Q2 (CD28(-)CD8(+)), respectively. Data from-two microarrays using RNA isolated from the adult cohort were analyzed as Q3 $(\mathrm{CD} 28(+) \mathrm{CD} 8(+))$ or Q4 (CD28(-)CD8(+)), correspondingly. Figure 3 schematically depicts the grouping of the eight circRNA microarrays and the four cross-group comparisons $(\mathrm{C} 1=\mathrm{Q} 1$ vs $\mathrm{Q} 2$; $\mathrm{C} 2=\mathrm{Q} 3$ vs $\mathrm{Q} 4$; $\mathrm{C} 3=\mathrm{Q} 1$ vs Q3; $\mathrm{C} 4=\mathrm{Q} 2$ vs Q4) that were performed (see Additional file 1: Table $S 2$ for the pooling and grouping protocol).

\section{Identification of the circRNA-miRNA connectivity}

Using microarray analysis we initially identified the circRNAs that exhibited a more than 2-fold change in expression. Then their respective miRNA targets, which

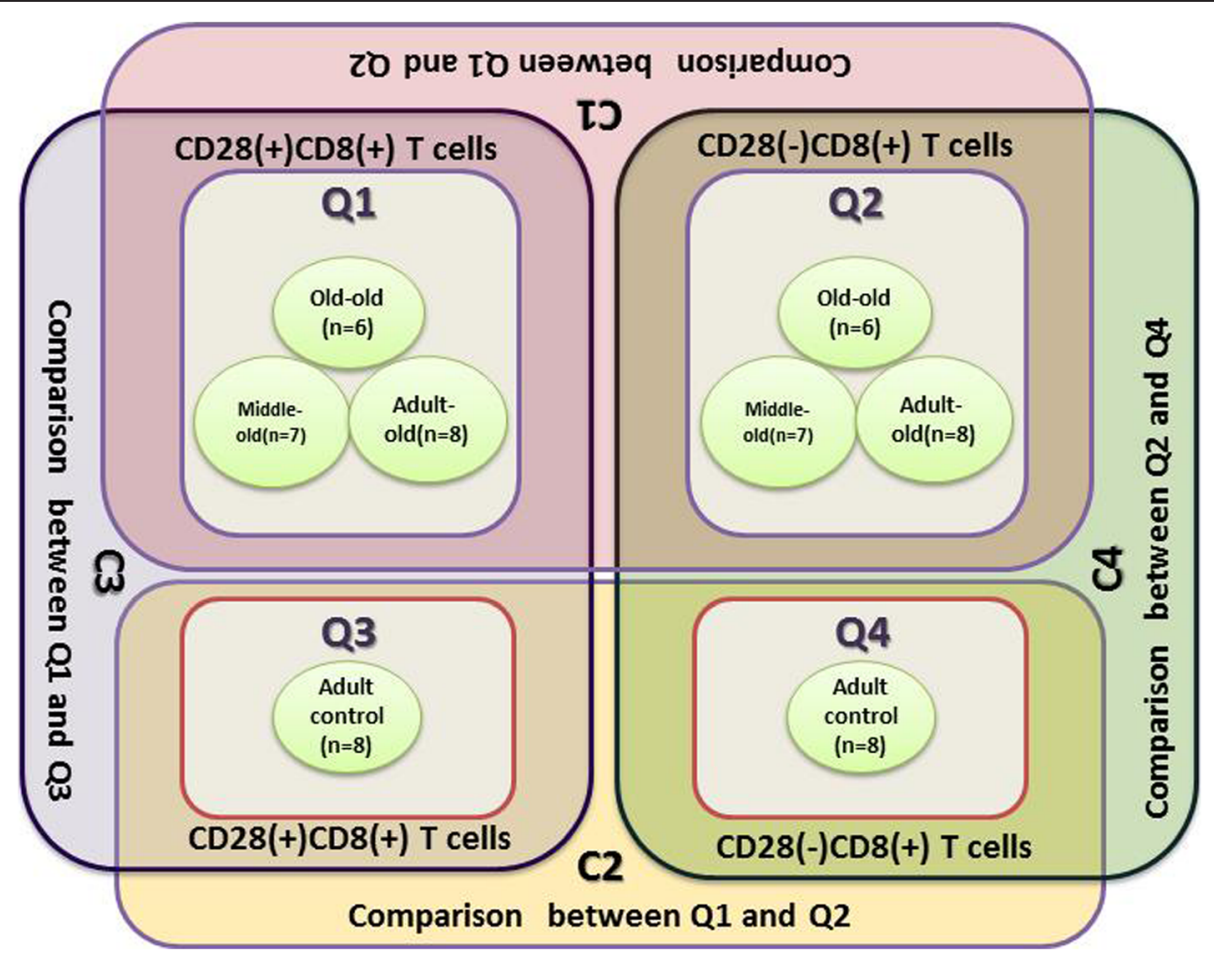

Fig. 3 Schematic strategy of pooling circRNA microarray profiles. Eight microarrays are depicted by 8 green circles. After eight microarray profiles were individually acquired, three microarray profiles detecting CD28(+)CD8(+)T cells from three age-dependent elderly subgroups were pooled as Q1,and three microarray profiles detecting CD28(-)CD8(+)T cells from three age-dependent elderly subgroups were pooled as Q2. Consequently, four cross-group comparisons were performed as: Comparison1 (C1:Q1 vs Q2 indicating CD28(+)CD8(+) vs CD28(-)CD8(+) T cells in the elderly); Comparison2 (C2: Q3 vs Q4 indicating CD28(+)CD8(+) vs CD28(-)CD8(+)T cells in the adult); Comparison 3 (C3:Q1 vs Q3 indicating CD28(+)CD8(+)T cells in the elderly vs in the adult); and Comparison4 (C4: Q2 vs Q4 indicating CD28(-)CD8(+)T cells in the elderly vs in the adult) 
were predicted based on sequence-pairing, were identified by TargetScan and verified by miRanda; thus the basic circRNA-miRNA connectivity was established. All subsequent biomathematical analyses were based on this basic molecular connectivity.

\section{Step-wise biomathematical circRNA candidate optimization}

After the basic circRNA-miRNA connectivity was determined, we focused our efforts on finding overlapping circRNA profiles between the $\mathrm{C} 1$ and $\mathrm{C} 4$ comparison groups. However, among the Top-10 circRNAs listed in Additional file 1: Table S1, none were commonly expressed in $\mathrm{C} 1$ and $\mathrm{C} 4$. Even when we expanded our list of candidates to include the Top-50 circRNAs, we observed no overlapping circRNA expression in $\mathrm{C} 1$ and C4. Therefore, we employed a step-wise biomathematical analysis to identify potential overlapping circRNAs (Animated details are depicted in Additional file 4: Online Supplemental PPT1).

First, to focus the targeted miRNA profile, the miRNA support vector regression (mirSVR) algorithm was used to score and rank the efficiency of the predicted microRNA targets (Betel et al. 2010). Accordingly, for each circRNA exhibiting greater than a 2-fold expression level, we identified 5miRNAs with the highest mirSVR (Top-5). Thus a "Top-5-mi" circRNA-miRNA network was established (one circRNA connecting to five miRNAs, miRNA-mediated Degree $=5$ ). Second, this Top-5 circRNA-miRNA network was re-ranked with respect to circRNA-mediated connectivity (one miRNA connecting to $\mathrm{N}$ circRNAs, circRNA-mediated Degree $=\mathrm{N}$ ). Then, circRNAs were ranked according to degree and the Top$10 \%$ candidates were identified for these restricted profiles. The cutoff of $10 \%$ was selected empirically according to our previous bioinformatics experience. Using these Top-10 \% circRNAs, a "re-enriched" Top-10 \% miRNAcircRNA network was established (“Top-10\%-mi"). Third, this Top-10 \% network was re-ranked according to the miRNA-mediated degree (one circRNA connecting $\mathrm{M}$ miRNAs,Degree $=M$ ). Our final investigation focused on circRNAs that exhibited degrees of 9 or greater in this reranked Top-10 \% network ("Top-10 \%-ci"). The selectedTop-9 circRNAs were subjected to Venny analysis to discern overlapping candidates (Table 1).

\section{Validation of candidate circRNAs using qRT-PCR}

Validation of circRNA was performed using quantitative polymerase chain reaction (qPCR; in triplicate) in three randomly selected RNA samples. Divergent primers (instead of commonly used convergent primers) were designed and optimized for eight circRNAs. The sequence of circRNA results were acquired from the database "circBase". The Glyceraldehyde 3-phosphate dehydrogenase (GAPDH) housekeeping gene was used as a control (Details are shown in Additional file 1: Table S5). To ensure the accuracy of the results, we verified the target specificity of the PCR primers using BLAST (http://blast.ncbi.nlm.nih.gov/Blast.cgi). The appearance of a single-peak in the melting curve indicated the specificity of the PCR results. The data were analyzed using the $\Delta \mathrm{Ct}$ method. Validation was designated by the ratio of the differential cellular expression/differential microarray expression.

\section{Annotation and function prediction for the validated circRNAs}

Validated candidate circRNAs were used as seeds to enrich a circRNA-miRNA-gene network according to the cutoff value determined using mirSVR. The predicted gene functions in the networks were annotated using Gene Ontology (GO) and KEGG Pathway using Database for Annotation, Visualization and Integrated Discovery (DAVID) [28].

\section{Statistical analysis}

Quantile normalization and subsequent data processing were performed using the R software package. All other statistical data were analyzed and visualized by GraphPad Prism5.0 (GraphPad Software, La Jolla, CA). The significance of qPCR validation of $\mathrm{C} 1$ and $\mathrm{C} 4$ were tested by Student's $t$ test $(P<0.05)$ and was considered statistically significant.

\section{Additional files}

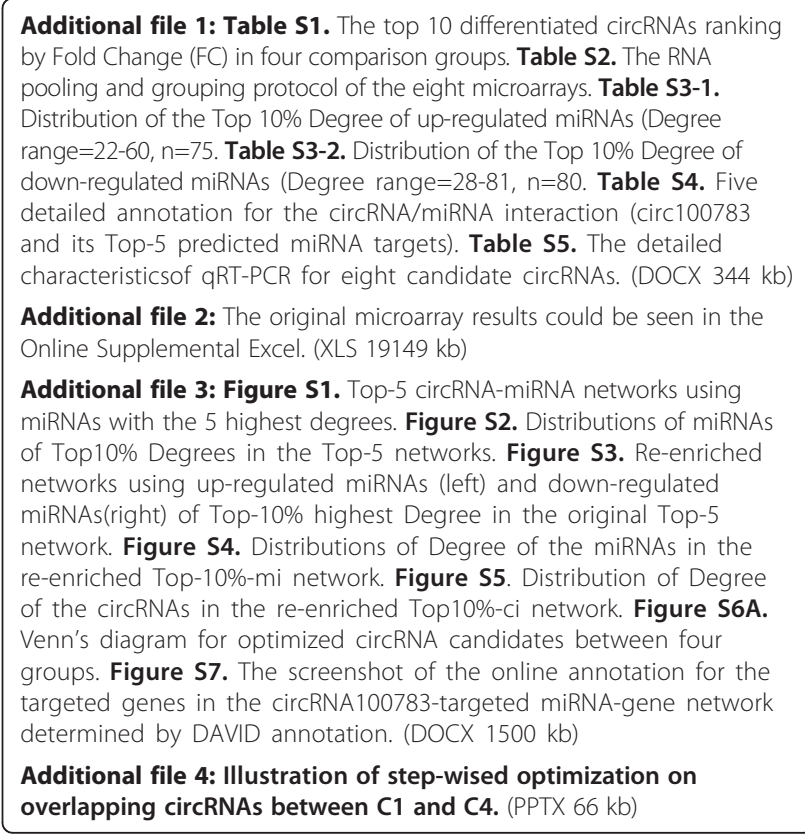

Competing interests

The authors declare that they have no competing interests. 


\section{Authors' contributions}

YHW carried out the GPCR and drafted the manuscript. XHY carried out flow cytometry and statistical analysis. SSL participated in clinical assessments and enrollment of the apparent healthy participants. $\mathrm{HH}$ participated in the design of the study, performed the bioinformatics analysis, and helped to draft the manuscript. All authors read and approved the final manuscript.

\section{Acknowledgements}

We thank Dr. Karl J. Franek for his professional editing.

\section{Funding}

The study was funded by The National Natural Science Foundation of China (No.81200689)

\section{Author details}

${ }^{1}$ Department of Geriatrics, First Affiliated Hospital of Harbin Medical University, Harbin 15001, China. ${ }^{2}$ First Institute of Geriatrics and Gerontology of Harbin Medical University, Harbin 15001, China. ${ }^{3}$ Department of Ophthalmology, First Affiliated Hospital of Harbin Medical University, Harbin 15001, China.

\section{Received: 16 July 2015 Accepted: 23 September 2015}

\section{Published online: 08 October 2015}

\section{References}

1. Weng NP, Akbar AN, Goronzy J. CD28(-) T cells: their role in the age-associated decline of immune function. Trends Immunol. 2009;30(7):306-12. doi:10.1016/j.it.2009.03.013.

2. Weinberger B, Welzl K, Herndler-Brandstetter D, Parson W, GrubeckLoebenstein B. CD28(-)CD8(+) T cells do not contain unique clonotypes and are therefore dispensable. Immunol Lett. 2009;127(1):27-32 doi:10.1016/j.imlet.2009.08.008.

3. Parish ST, Wu JE, Effros RB. Sustained CD28 expression delays multiple features of replicative senescence in human CD8 T lymphocytes. J Clin Immunol. 2010;30(6):798-805. doi:10.1007/s10875-010-9449-7.

4. Effros RB, Pawelec G. Replicative senescence of T cells: does the Hayflick Limit lead to immune exhaustion? Immunol Today. 1997;18(9):450-4

5. Monteiro J, Batliwalla F, Ostrer H, Gregersen PK. Shortened telomeres in clonally expanded CD28-CD8+ T cells imply a replicative history that is distinct from their CD28+CD8+ counterparts. J Immunol. 1996;156(10):3587-90

6. Khan N, Shariff N, Cobbold M, Bruton R, Ainsworth JA, Sinclair AJ, et al. Cytomegalovirus seropositivity drives the CD8 T cell repertoire toward greater clonality in healthy elderly individuals. J Immunol. 2002;169(4):1984-92.

7. Derhovanessian E, Maier AB, Hahnel K, Beck R, de Craen AJ, Slagboom EP, et al. Infection with cytomegalovirus but not herpes simplex virus induces the accumulation of late-differentiated CD4+ and CD8+ T-cells in humans. J Gen Virol. 2011;92(Pt 12):2746-56. doi:10.1099/vir.0.036004-0.

8. Koch S, Larbi A, Ozcelik D, Solana R, Gouttefangeas C, Attig S, et al. Cytomegalovirus infection: a driving force in human T cell immunosenescence. Ann N Y Acad Sci. 2007;1114:23-35. doi:10.1196/annals.1396.043.

9. Schwanninger A, Weinberger B, Weiskopf D, Herndler-Brandstetter D, Reitinger S, Gassner C, et al. Age-related appearance of a CMV-specific high-avidity CD8 + T cell clonotype which does not occur in young adults. Immun Ageing: I \& A. 2008:5:14. doi:10.1186/1742-4933-5-14.

10. Nilsson BO, Ernerudh J, Johansson B, Evrin PE, Lofgren S, Ferguson FG, et al. Morbidity does not influence the T-cell immune risk phenotype in the elderly: findings in the Swedish NONA Immune Study using sample selection protocols. Mech Ageing Dev. 2003;124(4):469-76.

11. Strindhall J, Nilsson BO, Lofgren S, Ernerudh J, Pawelec G, Johansson B, et al. No Immune Risk Profile among individuals who reach 100 years of age: findings from the Swedish NONA immune longitudinal study. Exp Gerontol. 2007:42(8):753-61. doi:10.1016/j.exger.2007.05.001.

12. Cao JN, Gollapudi S, Sharman EH, Jia Z, Gupta S. Age-related alterations of gene expression patterns in human CD8+ T cells. Aging Cell. 2010;9(1):19-31. doi:10.1111/j.1474-9726.2009.00534.x.

13. Fann M, Chiu WK, Wood 3rd WH, Levine BL, Becker KG, Weng NP. Gene expression characteristics of CD28null memory phenotype CD8+ T cells and its implication in T-cell aging. Immunol Rev. 2005;205:190-206. doi:10.1111/j.0105-2896.2005.00262.x.

14. Lazuardi L, Herndler-Brandstetter D, Brunner S, Laschober GT Lepperdinger G, Grubeck-Loebenstein B. Microarray analysis reveals similarity between CD8+CD28- T cells from young and elderly persons, but not of CD8+CD28+ T cells. Biogerontology. 2009;10(2):191-202. doi:10.1007/s10522-008-9167-1

15. Jeck WR, Sorrentino JA, Wang K, Slevin MK, Burd CE, Liu J, et al. Circular RNAs are abundant, conserved, and associated with ALU repeats. RNA 2013:19(2):141-57. doi:10.1261/rna.035667.112

16. Hansen TB, Jensen TI, Clausen BH, Bramsen JB, Finsen B, Damgaard CK, et al. Natural RNA circles function as efficient microRNA sponges. Nature. 2013;495(7441):384-8. doi:10.1038/nature11993.

17. Memczak S, Jens M, Elefsinioti A, Torti F, Krueger J, Rybak A, et al. Circular RNAs are a large class of animal RNAs with regulatory potency. Nature. 2013;495(7441):333-8. doi:10.1038/nature11928.

18. Wang PL, Bao Y, Yee MC, Barrett SP, Hogan GJ, Olsen MN, et al. Circular RNA is expressed across the eukaryotic tree of life. PLoS One. 2014;9(6):e90859. doi:10.1371/journal.pone.0090859.

19. Liang D, Wilusz JE. Short intronic repeat sequences facilitate circular RNA production. Genes Dev. 2014;28(20):2233-47. doi:10.1101/gad.251926.114.

20. Salzman J, Gawad C, Wang PL, Lacayo N, Brown PO. Circular RNAs are the predominant transcript isoform from hundreds of human genes in diverse cell types. PLoS One. 2012;7(2):e30733. doi:10.1371/journal.pone.0030733.

21. Westholm JO, Miura P, Olson S, Shenker S, Joseph B, Sanfilippo P, et al. Genome-wide analysis of drosophila circular RNAs reveals their structura and sequence properties and age-dependent neural accumulation. Cell reports. 2014;9(5):1966-80. doi:10.1016/j.celrep.2014.10.062.

22. Vescovini R, Telera A, Fagnoni FF, Biasini C, Medici MC, Valcavi P, et al. Different contribution of EBV and CMV infections in very long-term carriers to age-related alterations of CD8+ T cells. Exp Gerontol. 2004;39(8):1233-43. doi:10.1016/j.exger.2004.04.004.

23. Kuparinen T, Marttila S, Jylhava J, Tserel L, Peterson P, Jylha M, et al. Cytomegalovirus (CMV)-dependent and -independent changes in the aging of the human immune system: a transcriptomic analysis. Exp Gerontol. 2013;48(3):305-12. doi:10.1016/j.exger.2012.12.010.

24. Salzman J, Chen RE, Olsen MN, Wang PL, Brown PO. Cell-type specific features of circular RNA expression. PLoS Genet. 2013;9(9):e1003777. doi:10.1371/journal.pgen.1003777.

25. Guo JU, Agarwal V, Guo H, Bartel DP. Expanded identification and characterization of mammalian circular RNAs. Genome Biol. 2014;15(7):409. doi:10.1186/s13059-014-0409-z.

26. Thomas LF, Saetrom P. Circular RNAs are depleted of polymorphisms at microRNA binding sites. Bioinformatics. 2014;30(16):2243-6. doi:10.1093/bioinformatics/btu257.

27. Wang YH, Jiang YP, Luo SS, Qiao FF, Han H. Multilevel model estimation of age-dependent individual-specific trajectories for left ventricular echocardiographic indexes in an asymptomatic elderly cohort. J Appl Physiol. 2012;112(7):1128-34. doi:10.1152/japplphysiol.01256.2011.

28. da Huang W, Sherman BT, Lempicki RA. Systematic and integrative analysis of large gene lists using DAVID bioinformatics resources. Nat Protoc 2009:4(1):44-57. doi:10.1038/nprot.2008.211.

\section{Submit your next manuscript to BioMed Central and take full advantage of:}

- Convenient online submission

- Thorough peer review

- No space constraints or color figure charges

- Immediate publication on acceptance

- Inclusion in PubMed, CAS, Scopus and Google Scholar

- Research which is freely available for redistribution 